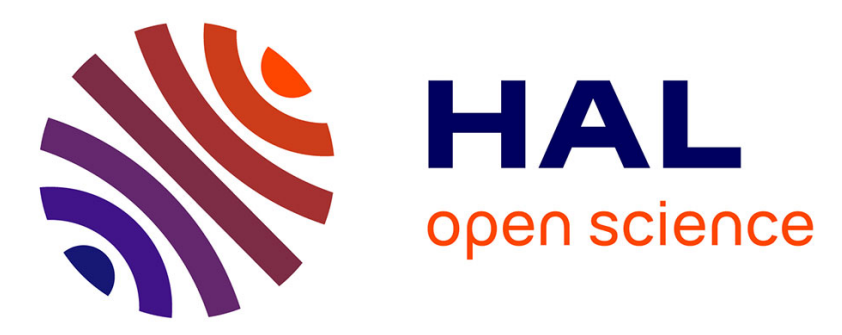

\title{
Electrostatically-induced modal crosstalk phenomena in resonant MEMS sensors
}

\author{
Alexis Brenes, Jérôme Juillard, Filipe Vinci dos Santos
}

\section{To cite this version:}

Alexis Brenes, Jérôme Juillard, Filipe Vinci dos Santos. Electrostatically-induced modal crosstalk phenomena in resonant MEMS sensors. 2014 Symposium on Design, Test, Integration and Packaging of MEMS/MOEMS (DTIP) , Apr 2014, Cannes, France. 10.1109/DTIP.2014.7056659 . hal-01086397

\section{HAL Id: hal-01086397 \\ https://hal-centralesupelec.archives-ouvertes.fr/hal-01086397}

Submitted on 6 Jan 2015

HAL is a multi-disciplinary open access archive for the deposit and dissemination of scientific research documents, whether they are published or not. The documents may come from teaching and research institutions in France or abroad, or from public or private research centers.
L'archive ouverte pluridisciplinaire HAL, est destinée au dépôt et à la diffusion de documents scientifiques de niveau recherche, publiés ou non, émanant des établissements d'enseignement et de recherche français ou étrangers, des laboratoires publics ou privés. 


\section{Electrostatically-induced modal crosstalk phenomena in resonant MEMS sensors}

\author{
A. Brenes \\ THALES Avionics \\ Valence, France \\ alexis.brenes@supelec.fr
}

\author{
J. Juillard ${ }^{1}$ and F. Vinci dos Santos $^{2}$ \\ E3S ${ }^{1}$ and THALES Chair ${ }^{2}$ \\ Ecole Supérieure d'Electricité (Supélec) \\ Gif-sur-Yvette, France
}

\begin{abstract}
The aim of this paper is to introduce an explanation for the amplitude saturation and inhibition phenomena in resonant MEMS pressure sensors, via the electrostatic coupling of two resonance modes. Our analysis and experimental results reveal that these phenomena may be ubiquitous in electrostatic resonant MEMS sensors.
\end{abstract}

\section{Keywords : Autoparametric resonance, electrostatic nonlinearity.}

\section{INTRODUCTION}

In this paper we introduce, analyze and give experimental evidence of electrostatically-induced power-handling limiting phenomena in resonant MEMS, consequently to the coupling of two vibration modes. Previous work has addressed such coupling under more constrained conditions and hypothesis. For example, [1] has shown that autoparametric resonance may be induced by the existence of purely mechanical nonlinear coupling between two resonance modes, while [2] deals with electrostatically-induced phenomena for single degree-offreedom (dof) structures. Furthermore, the mentioned references address open-loop excitation of the structure, under the restriction of small displacement amplitudes with respect to the electrostatic gap. In contrast, our research targets a two-dof system consisting of two linearly-coupled resonators, one of which is capacitively sensed and actuated in closed-loop, and we develop transient regime simulations valid for displacements comparable to the gap. Our qualitative analysis is supported by our experimental results, suggesting that the observed phenomena may be ubiquitous in electrostatic resonant MEMS sensors.

\section{EXPERIMENTAL SETUP}

The diaphragm-based silicon pressure sensor described in this paper was originally developed by Sextant Avionics (currently THALES Avionics) [3] and is industrially assembled by the fusion-bonding of three etched silicon wafers (see Fig. 1). It consists of a resonant beam resting on a rectangular diaphragm which deflects under pressure. During the manufacturing process, the beam is encapsulated in vacuum in order to achieve a high mechanical Q-factor $(\approx 20000)$. The resonance frequency $f_{0}$ of the device is close to $65 \mathrm{kHz}$.

In our set-up, the sensing cell is placed in a closed-loop oscillator in which resonance oscillations are maintained by electrostatic pulses [4], using an analog feedback loop consisting of a charge amplifier, a comparator and a differentiator (Fig. 2). The motional current is integrated in the charge amplifier and converted into an output voltage. Hence, the output voltage is the image of the mechanical motion of the resonator (distorted by the capacitive detection nonlinearity). The output voltage is used as an input to a comparator and then differentiated through a bias tee. The components of the bias tee are chosen so that its cutoff frequency is much larger than $f_{0}$. Thus, the square output of the comparator is differentiated, which results in positive and negative voltage pulses that are triggered when the output voltage crosses 0 [5]. A typical output signal is shown in Fig. 3. Note that, due to the one-sided detection scheme used in the sensor, the output waveform is asymmetrical, increasingly more as the oscillation amplitude rises, and the negative peak voltage $|V-|$ is always greater (in magnitude) than the positive peak voltage $V+$. Such an actuation scheme is theoretically known for providing a larger travel range than sine-wave or square-wave actuation [6]. Considering the capacitive actuation and detection scheme, the output signal exhibits positive and negative voltage pulses, superposed on the motional signal (Fig. 3).

With this setup, a wide range of operating conditions can be explored by changing the driving level (pulse amplitude $V_{a c t}$ ) and the bias voltage $V_{b}$. Typical experimental results are shown in Fig. 4. We note that increasing the bias conditions beyond a certain level often results in a counterintuitive saturation of the oscillation amplitude, possibly after an amplitude jump (for large values of $V_{a c t}$ ).

\section{ANALYSIS}

Saturation phenomena in closed-loop MEMS oscillators may be caused by nonlinear modal interactions [1]. To determine through which mechanism these interactions occur, a finite element analysis of the structure was performed. The first two resonance modes are represented in Fig. 5 and Fig. 6. It was found that the first eigenfrequency is close to the experimentally-observed frequency. The second mode (diaphragm bending mode) also appears to have an eigenfrequency slightly inferior to $2 f_{0}$ (Fig. 7) over the whole operating range of the sensor. Due to the electrostatic softening non-linearity, $f_{0}$ should decrease (i) when increasing the bias voltage and (ii) when increasing the oscillation amplitude, whereas the second eigenfrequency should be largely unchanged. Thus, the 1:2 ratio between the two frequencies might be reached and modal interaction may be induced. This analysis underlines the importance of considering modal 
interactions for this kind of pressure sensors.

To verify our hypothesis that electrostatic nonlinearity is behind the observed saturation/inhibition phenomena, a simple model, amenable to analysis, of the system was put together (Fig. 8). This model corresponds to the one-sided electrostatic actuation and detection of a flat, rigid structure with a massive, elastic foundation - a surrogate model of structures such as resonant pressure sensors or accelerometers, in which a resonant strain gauge is used to sense the deformation of a diaphragm (Fig. 1) or the displacement of a seismic mass. Considering the natural frequencies given by finite-element simulations, we aimed to analyze the large-amplitude dynamics of the system through nonlinear transient simulations. We chose this approach to make it possible to explore the variety of behaviors exhibited by such systems.

The following equations correspond to the mechanical model describing the behavior of the sensor:

$$
\left\{\begin{array}{l}
\omega_{a}^{2} a+\frac{\omega_{a}}{Q_{a}} \frac{d a}{d t}+\frac{d^{2} a}{d t^{2}}=\omega_{a}^{2} \eta\left(\frac{1+v(t)}{1-a}\right)^{2}+\omega_{a}^{2} b \\
\left(\omega_{b}^{2}+\mu \omega_{a}^{2}\right) b+\frac{\omega_{b}}{Q_{b}} \frac{d b}{d t}+\frac{d^{2} b}{d t^{2}}=\mu \omega_{a}^{2} a+\omega_{b}^{2} f_{e x t}
\end{array}\right.
$$

In this model, $G$ is the gap between the two electrodes and $a=z_{a} / \mathrm{G}$ and $b=z_{b} / \mathrm{G}$ are respectively the dimensionless displacement of the beam and the diaphragm. $\omega_{a}$ and $\omega_{b}$ are the natural frequencies of the corresponding modes, $Q_{a}$ and $Q_{b}$ their quality factors and $\mu=m_{a} / m_{b}$ is the ratio of the two masses. $\eta \propto V_{b}^{2}$ is an electromechanical coupling coefficient, $f_{\text {ext }}$ corresponds to the dimensionless force applied on the diaphragm, $v$ to the ratio $V_{a c t} / V_{b}$. Moreover, the electrostatic pulses are calculated via a model of the electronic feedback loop based on ideal components. This model can be simulated with Matlab/Simulink.

The results obtained when performing a 1s-sweep of the bias voltage from $25 \mathrm{~V}$ to $65 \mathrm{~V}$ are reported in Fig. 9, for different values of $V_{\text {act }}$. The values of the model parameters are: $\omega_{a}=4 \times 10^{5} \mathrm{rad} . \mathrm{s}^{-1}, \quad \omega_{b}=7.1 \times 10^{5} \mathrm{rad} . \mathrm{s}^{-1}, \quad Q_{a}=2 \times 10^{4}, \quad Q_{b}=150$, $\mu=0.15, f_{\text {ext }}=0.15, \eta=4 / 27 V_{b}^{2} / V_{p i}{ }^{2}$ where $V_{p i}$ is chosen equal to $200 \mathrm{~V}$. For this set of parameters, amplitude saturation and inhibition appears as in experimental results, around the same values of $V_{b}$.

\section{DISCUSSION}

There is a good qualitative agreement between the experimental results and those predicted by the lumped model of Fig. 8, suggesting that the saturation phenomenon is caused by the coupling between the beam and the diaphragm. The simulations show that these counterintuitive variations of oscillation amplitude and frequency correspond to the appearance of frequency-locked states in which the structure and the foundation are both close to resonance, respectively at $\omega \approx \omega_{a}$ and $2 \omega \approx \omega_{b}$, and in which the small terms at $2 \omega$ are dramatically amplified by the electrostatic nonlinearity and inhibit the principal resonance.

Note that two other causes may be suspected to induce amplitude inhibition. The best-known one is autoparametric inhibition via the Duffing nonlinearity [1]. However, further simulations show that accounting for a nonlinear (cubic) coupling between the two masses cannot explain the observed behavior. The other plausible cause is linked to the triggering of the pulses. Because of the one-sided capacitive detection scheme, the output voltage of the charge amplifier is asymmetrical, as already mentioned, which results in a comparator output with a duty cycle smaller than $50 \%$. Hence, the pulses are not exactly generated when the beam passes through its equilibrium position, but when the distorted output voltage crosses 0 (or, equivalently, when the instantaneous charge is equal to its average value). Simulations show that this phenomenon cannot be the major cause of 1:2 parametric resonance, but rather that it acts as a second-order correction and tends to accentuate the saturation and inhibition induced by modal coupling. Finally, it should be pointed out that the observed behavior cannot be explained by autoparametric resonance of a single-dof system, as reported in [2].

The discrepancies remaining between our simulated and experimental results may be explained by couplings involving more than two modes and by accounting for beam deformation, which is the subject of ongoing work. A further steady-state analysis of (1) associated to an optimization procedure should also allow us to extract more accurate parameter values for our model, and thus to improve the fit between simulation and experiments.

\section{CONCLUSION}

The original analysis and results presented in this paper may be used for qualitatively explaining the power handling limits of resonant MEMS driven at large oscillation amplitudes and help avoiding the accidental occurrence of autoparametric resonance phenomena.

The modal interaction proposed in this paper is allowing the device to work unexpectedly well with large amplitude oscillations, and it paves the way to new large amplitude operating regimes with larger SNR. The development of nonlinear testing modes for resonant sensor calibration, of binary sensing modes (as suggested in [2]) or resonant switches can also be considered.

\section{REFERENCES}

[1] C. van der Avoort et al, "Amplitude saturation of MEMS resonators explained by autoparametric resonance," J. Micromech. Microeng., vol. 20, no. 10, 15 pp., Oct. 2010.

[2] J.F. Rhoads et al., "Generalized parametric resonance in electrostatically actuated microelectromechanical oscillators," J. Sound and Vibration, vol. 296, pp. 797-829, Oct. 2006.

[3] J. Mandle, O. Lefort and A. Migeon, "A new micromachined silicon high-accuracy pressure sensor," Sensors and Actuators A, vol. 46, no. 1-3, pp. 129-132, 1995.

[4] E. Colinet, J. Juillard and S. Guessab, "Actuation of resonant MEMS using short pulsed forces," Sensors and Actuators A, vol. 115, no. 1, pp. 118$125,2004$.

[5] J. Juillard, A. Bonnoit, E. Avignon, S. Hentz and E. Colinet, "Large amplitude dynamics of micro/nanomechanical resonators actuated with electrostatic pulses, ” Journal of Applied Physics A, vol. 107, no. 1, 2010.

[6] J. Juillard, G. Arndt, J. Arcamone and E. Colinet, "Influence of excitation waveform and oscillator geometry on the resonant pull-in of capacitive MEMS oscillators," in Proc. of DTIP, Barcelona, Sp., 2013, pp. 1-4. 


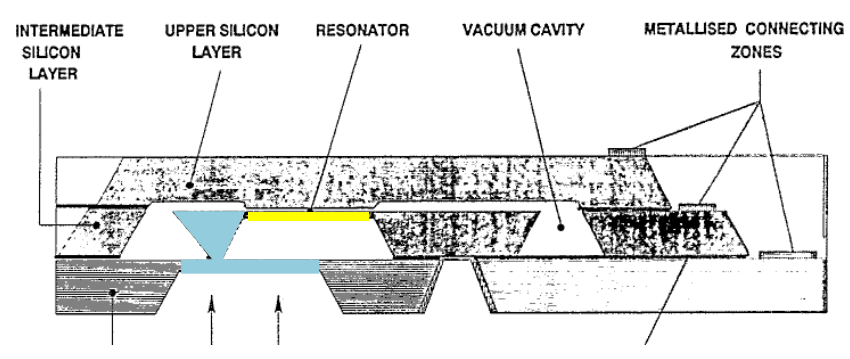

Fig. 1 - Sensor structure (from [3]). The sensing element consists in a resonant silicon beam encapsulated in vacuum, with a massive stud bonded to a silicon diaphragm. The beam is capacitively actuated and sensed with the facing electrode.

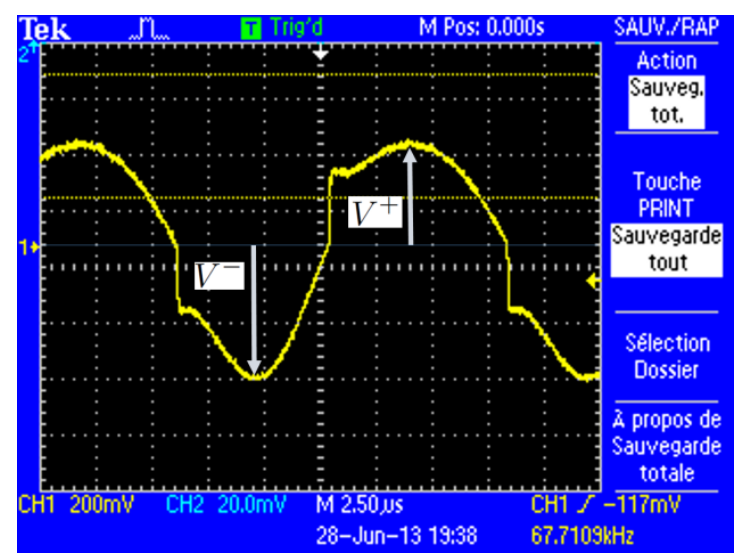

Fig. 3 - Shape of the output voltage. The output is the image of the mechanical motion of the resonator. Note that the pulses used for actuating the structures are visible because of capacitive feedthrough.

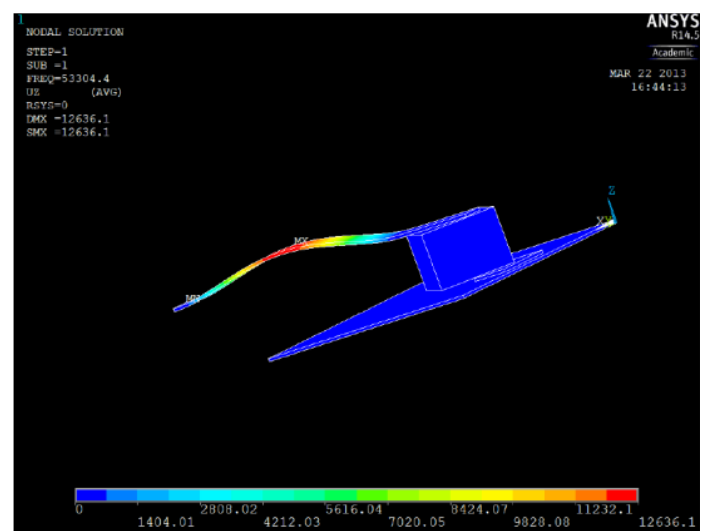

Fig. 5 - FEM Modal analysis - Shape of the first mode.

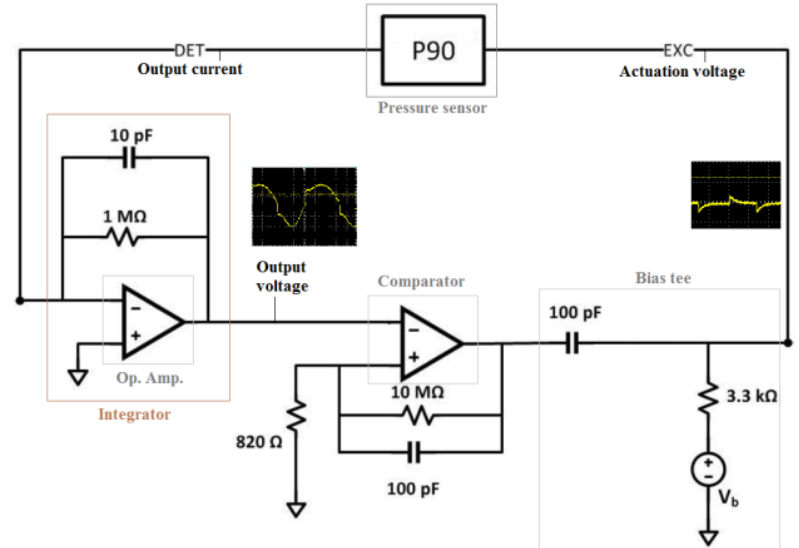

Fig. 2 - Electrical circuit - Closed-loop system.

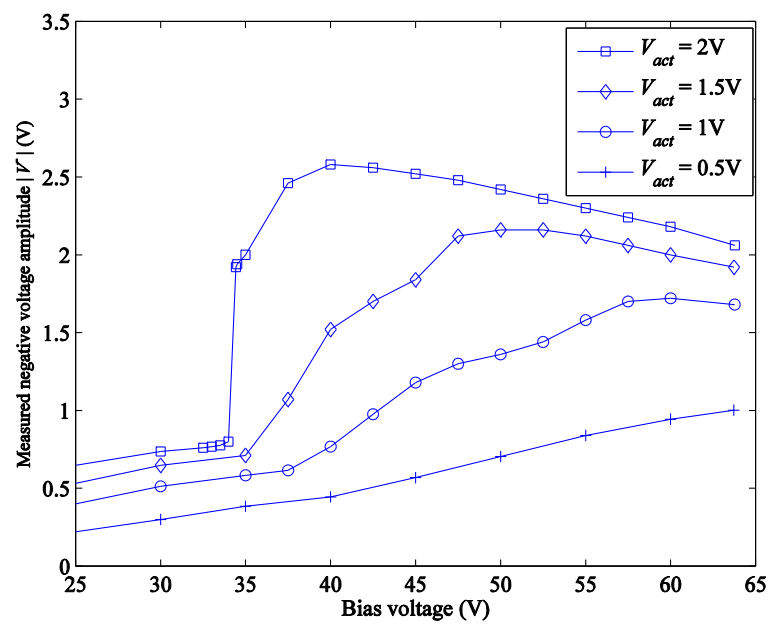

Fig. 4 - Experimental results for a set of $V_{a c t}-$ Negative voltage amplitude $\left|V^{-}\right|$vs. bias voltage.

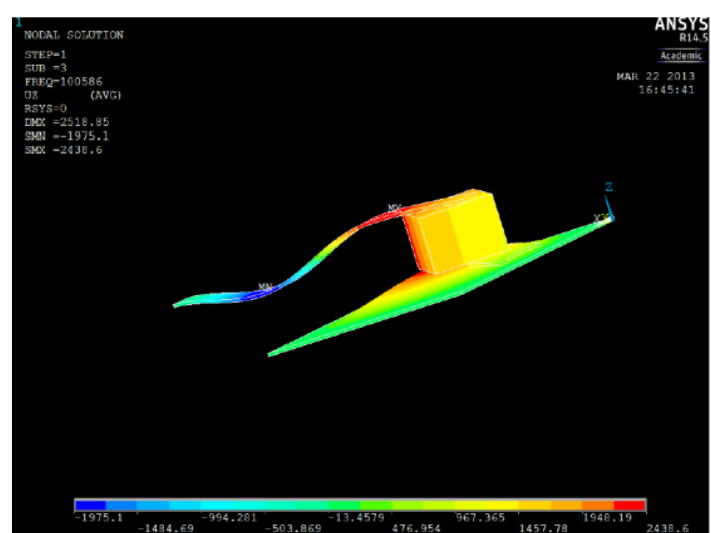

Fig. 6-FEM Modal analysis - Shape of the second mode. 


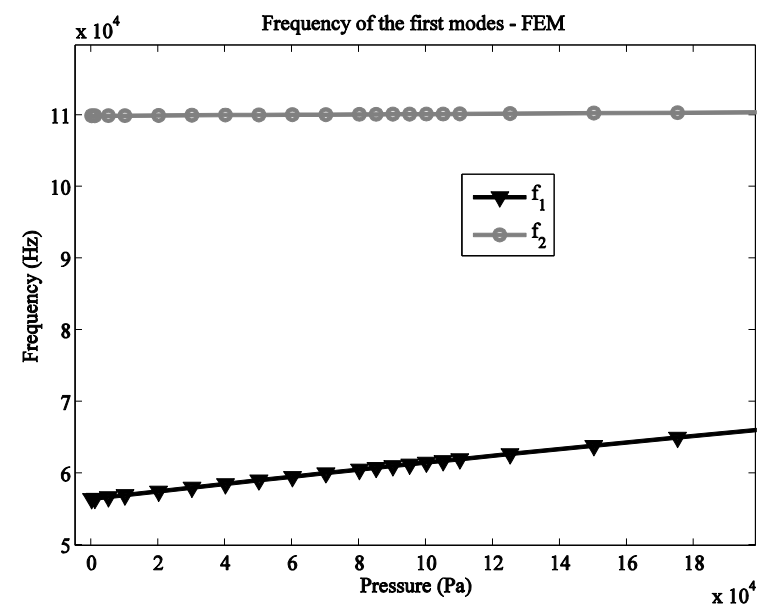

Fig. 7 - Natural frequencies of the beam (black line) and diaphragm (grey line) modes - FEM analysis - Evolution vs. pressure. In the whole operating range of the sensor, from $0 \mathrm{~Pa}$ to $1.4 \mathrm{hPa}$, the ratio between the two frequencies is close to 2 .

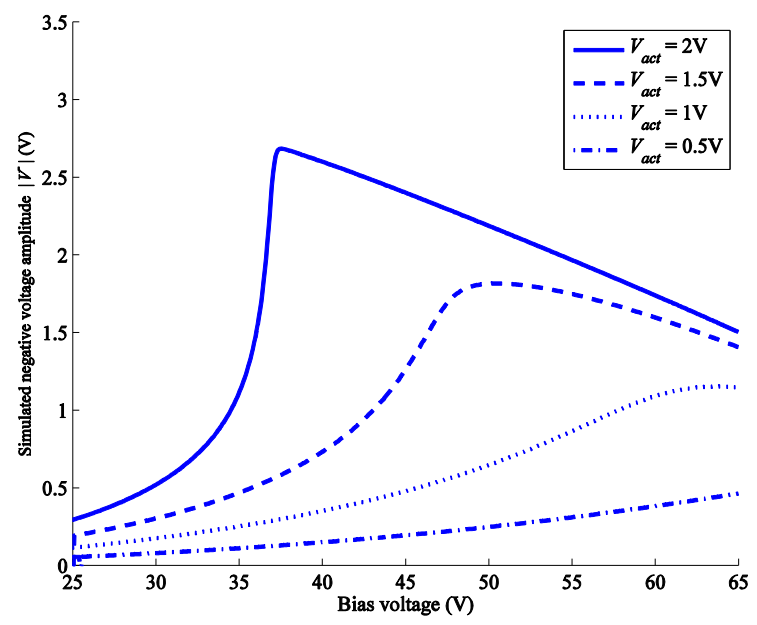

Fig. 9 - Simulated results for the same set of $V_{\text {act }}$ as Fig. 4.

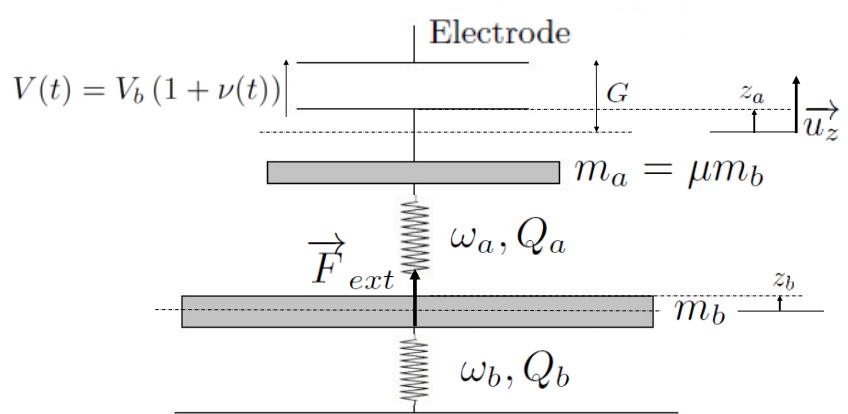

Fig. 8 - Idealized model of an electrostatic resonant sensor consisting of a resonant gauge with mass $m_{a}$ on an elastic foundation with mass $m_{b} . F_{\text {ext }}$ is an external force acting on the foundation, caused by a pressure differential, for example. 